\title{
Transport Theory in the Context of the Normalized Generalized Statistics
}

\author{
F. Q. Potiguar and U. M. S. Costa \\ Universidade Federal do Ceará, Departamento de Física, Campus do Pici, 60455-760 Fortaleza, Ce, Brazil
}

(October 24, 2018)

\begin{abstract}
In this work assuming valid the equipartition theorem and using the normalized q-expectation value, we obtain, until first order approximation, the hydrodynamics equation for the generalized statistics. This equations are different from those obtained in the context of the Boltzmann-Gibbs statistics. This difference is that now appears two transport coefficient that depend on the q-value.
\end{abstract}

PACS number(s): 05.20.-y, 05.20.Jj, 05.20.Gg

\section{INTRODUCTION}

The Generalized Statistics was first proposed by Tsallis [1] in 1988. It has attracted a lot of attention lately for it has been well applied to many systems, like fully developed turbulence [2] and self-graviting systems [3]. For a periodically updated list please see reference [4]. This generalization is based on the new entropic form, namely:

$$
S_{q}=k_{B} \frac{1-\sum_{i=1}^{W} p_{i}^{q}}{1-q}
$$

where $k_{B}$ is the Boltzmann constant, $p_{i}$ is the probability that the system is in a state labeled by $i, q$ is a real parameter and the sum runs over all the allowed states. This entropy has the essential feature that it is non-extensive (non-additive). For two independent systems,i.e., for two systems which have independent probabilities: $p^{(A U B)}=p^{(A)} p^{(B)}$, the entropy of the whole system is written as:

$$
S_{q}^{(A U B)}=S_{q}^{(A)}+S_{q}^{(B)}+(1-q) S_{q}^{(A)} S_{q}^{(B)}
$$

At that time it was used to calculate the physical quantities average values without proper normalization. Later Tsallis, Mendes and Plastino [5] have introduced a new form of calculating expectation values, the so called normalized q-expectation value. Let $O$ be some physical observable, its expectation value, in this context, is given by:

$$
<O>_{q}=\frac{\sum_{i=1}^{W} p_{i}^{q} O_{i}}{\sum_{i=1}^{W} p_{i}{ }^{q}}
$$

where:

$$
p_{i}=\left[1-(1-q) \beta\left(H_{i}-U_{q}\right)\right]^{\frac{1}{1-q}}
$$

is the canonical ensemble distribution function, with $U_{q}$ being the q-average internal energy known, a priori, from experiment.

Tsallis introduced a cut-off condition [1] in this formalism. It says that if the argument of the distribution function is negative, this distribution vanishes, in other words, if $1-(1-q) \beta\left(H_{i}-U_{q}\right)<0, p_{i}=0$.

In 1999, Boghosian [8] has obtained the hydrodynamic equations for the generalized statistics. He calculated them using the unnormalized q-expectation value and the well known Chapman-Enskog expansion. He showed that the equation of state and the internal energy for the ideal gas are q-dependent. He showed also that, in first order approximation, the conservation equations don't change and, in second order approximation, only the energy conservation equation change.

The first change is that the thermal conductivity coefficient is now q-dependent. It is given by, in the threedimensional case:

$$
K_{B}=\frac{1}{A_{q}+(1-q)} \frac{5 \tau \rho}{3 m \beta}
$$

where:

$$
A_{q}=1+(1-q) \frac{3}{2}
$$

$\tau$ is the expansion parameter, $\rho$ is the mass density of the system and $\beta$ is the inverse of the temperature.

There was, also, the appereance of a new term in this equation, a gradient of pressure, with a new transport coefficient, which was called anomalous transport coefficient:

$$
T_{q}=\frac{1-q}{A_{q}\left[A_{q}+(1-q)\right]} \frac{5 \tau \rho}{3 m \beta}
$$

Obviously this coefficient vanishes in the extensive limit $q \rightarrow 1$.

We propose in this paper to obtain the hydrodynamic equations using a different method and using the normalized q-expectation value. We'll see that these new features will led us to the correct equation of state for the ideal gas and to different dependence on $q$ of the transport coefficient. In section two we obtain the Conservation Theorems for the conserved quantities in molecular collisions, we make the zero order approximation, that corresponds to the first order approximation in Boghosian [8] calculations and we present the first order approximation (Boghosian second order approximation). 
Finally we presents our results and conclusion in section three.

\section{NORMALIZED HYDRODYNAMIC EQUATIONS}

\section{A. Conservation Theorems}

Here we present our way to obtain the hydrodynamic equations. We'll follow reference [10] and assume the energy equipartition theorem to be valid [7]. We are studying an ideal gas in the 6-dimensional phase space. The one particle hamiltonian for this system reads:

$$
H=\frac{m}{2} U^{2}
$$

where $U_{i}=v_{i}-u_{i}$, with $u_{i}=<v_{i}>$ the average velocity and $\left\langle U_{i}\right\rangle=0, m$ stands for the mass of the molecule and the index $i$ runs from 1 to 3 and indicates the components of the velocity.

It consists in obtaining the equations which give the transport of the molecular quantities that are conserved in a molecular collision. To do this, we assume first that we are dealing only with binary collision and that the two colliding molecules are labeled 1 and 2 . We are studying only the molecule 1 . We also assume that the molecular chaos is still valid.

To obtain the conservation theorems we consider the Boltzmann transport equation:

$$
\begin{gathered}
\left(\frac{\partial}{\partial t}+v_{i} \partial_{i}+\frac{F_{i}}{m} \partial_{v_{i}}\right) F_{1}= \\
\int d^{3} v_{2} d^{3} v_{f_{1}} d^{3} v_{f_{2}} \delta^{4}\left(V_{f}-V_{i}\right)\left|T_{f i}\right|^{2}\left(F_{f_{2}} F_{f_{1}}-F_{2} F_{1}\right)
\end{gathered}
$$

where $v_{i}$ are the components of the thermal velocity of the molecules, $F_{i}$ are the components of the external forces, which are considered here velocity independent, the index $2, f_{1}, f_{2}$ refers to the second molecule before the collision and to the both molecules after the collision, the 4-dimensional delta function and the transition matrix elements $T_{f i}$ are related to the differential scattering cross section of the collision and the term between parenthesis is the molecular chaos assumption. $F_{1}$ is the particle density which is the solution for (8) and is unknown.

The equilibrium distribution function is given by the escort distribution:

$$
F_{q}=n \frac{\left[1-\frac{1-q}{A_{q}} \beta H\right]^{\frac{q}{1-q}}}{\int_{-\infty}^{+\infty}\left[1-\frac{1-q}{A_{q}} \beta H\right]^{\frac{q}{1-q}} d^{3} U}
$$

where $\beta$ is the inverse of the temperature $\beta=\frac{1}{k_{B} \beta}$. The factor $n$ is the particle density. It appears here because this distribution is described in the one particle phase space, so it is not simply a probability distribution, it is the number of particles in the system which have position between $\vec{x}$ and $\vec{x}+d \vec{x}$ and velocity between $\vec{v}$ and $\vec{v}+d \vec{v}$. This function is related to the escort probability distribution through the relation $F_{q}=n P_{q}$. The normalization condition reads $\int F_{q} d^{3} U=n$.

We define a collision conserved quantity $\chi(\vec{x}, \vec{v}, t)$, which have the conservation property $\chi_{1}+\chi_{2}=\chi_{f_{1}}+\chi_{f_{2}}$. The following theorem can be proved:

$$
\int d^{3} v_{1} \chi_{1}(\vec{x}, \vec{v})\left(\frac{\partial F_{1}}{\partial t}\right)_{\text {coll }}=0
$$

where $\left(\frac{\partial F_{1}}{\partial t}\right)_{\text {coll }}$ is the collisional integral, making the changing of variables below:

First: $\vec{v}_{1} \rightarrow \vec{v}_{2}$ and vice-versa.

Second: $\vec{v}_{1} \rightarrow \vec{v}_{f_{1}}, \vec{v}_{2} \rightarrow \vec{v}_{f_{2}}$ and vice-versa.

Third: $\vec{v}_{1} \rightarrow \vec{v}_{f_{2}}, \vec{v}_{2} \rightarrow \vec{v}_{f_{1}}$ and vice-versa.

As these changes don't affect the transition matrix elements $T_{f i}$ and the delta function, we add each of the integrals obtained with equation (10)and divide the result by 4 . We end up with:

$$
\begin{gathered}
\int d^{3} v \chi\left(\frac{\partial F_{1}}{\partial t}\right)_{c o l}=\frac{1}{4} \int d^{3} v_{1} d^{3} v_{2} d^{3} v_{f_{1}} d^{3} v_{f_{2}} \delta^{4}\left(P_{f}-P_{i}\right) \\
\times\left|T_{f i}\right|^{2}\left(F_{f_{2}} F_{f_{1}}-F_{2} F_{1}\right)\left(\chi_{1}+\chi_{2}-\chi_{f_{1}}-\chi_{f_{2}}\right)
\end{gathered}
$$

Through the conservation of $\chi_{1}$, the theorem is proved. Using equation (8), we have, changing the volume element form $d^{3} v$ to $d^{3} U$ :

$$
\int d^{3} U_{1} \chi_{1}\left(\frac{\partial}{\partial t}+v_{i} \partial_{i}+\frac{F_{i}}{m} \partial_{v_{i}}\right) F_{1}(\vec{x}, \vec{v}, t)=0
$$

Defining the expectation value as:

$$
<O>=\frac{1}{n} \int F_{1} O d^{3} U
$$

and rearranging terms in (11), we have the desired conservation theorem:

$$
\begin{gathered}
\frac{\partial}{\partial t}<n \chi_{1}>+\partial_{i}<n v_{i} \chi_{1}>-n<v_{i} \partial_{i} \chi_{1}> \\
-\frac{n}{m} F_{i}<\partial_{v_{i}} \chi_{1}>=0
\end{gathered}
$$

Now we calculate the above equation for the three conserved quantities, namely mass, momentum and energy. For the mass, $\chi_{1}=m$, we have:

$$
\frac{\partial \rho}{\partial t}+\partial_{i}\left(\rho u_{i}\right)=0
$$

where:

$$
\rho=m n(\vec{x}, t)
$$


is the mass density of the system.

For the momentum, $\chi_{1}=m v_{i}$, we get:

$$
\frac{\partial}{\partial t}\left(\rho u_{i}\right)+\partial_{j}\left(\rho<U_{i} U_{j}>\right)+\partial_{j}\left(\rho u_{i} u_{j}\right)=\rho \frac{F_{i}}{m}
$$

Finally for the energy, $\chi_{1}=\frac{m}{2} U^{2}$, we have, using the mass conservation equation (14):

$$
\begin{gathered}
\frac{\partial}{\partial t}\left(\frac{1}{\beta}\right)+u_{i} \partial_{i}\left(\frac{1}{\beta}\right) \\
+\frac{2 m \rho}{3} \partial_{i} u_{j}<U_{i} U_{j}>+\frac{m}{3} \partial_{i}\left(\rho<U_{i} U^{2}>\right)=0
\end{gathered}
$$

where we used the fact that the internal energy for this system is given by the classical energy equipartition theorem. All we have to do now is calculate the two expectation values in order to obtain the hydrodynamic equation for the generalized statistics.

\section{B. The Zero Order Approximation}

To obtain the exact equations, we should use the solution for (8) $F_{1}$. As we don't still have this solution, we must approximate it. We assume that the system, locally, obeys equation (9). This is the local equilibrium assumption. We impose a space and time dependence of the macroscopic parameters number density $n$, average velocity $u_{i}$ and temperature $\frac{1}{\beta}$. Hence the distribution function reads:

$$
F_{q}{ }^{(0)}=n \frac{\left[f^{(0)}\right]^{\frac{q}{1-q}}}{\int\left[f^{(0)}\right]^{\frac{q}{1-q}} d^{3} U}=\frac{n}{I}\left[f^{(0)}\right]^{\frac{q}{1-q}}
$$

The integral in the denominator is readily calculated:

$$
I=\int\left[1-\frac{1-q}{A_{q}} \beta \frac{m}{2} U^{2}\right]^{\frac{q}{1-q}} d^{3} U
$$

Using the spherical volume element and making the following change of variable:

$$
U=\left(\left|\frac{A_{q}}{1-q}\right| \frac{2}{m \beta}\right)^{1 / 2} x^{1 / 2}
$$

we have:

$$
I=2 \pi\left(\left|\frac{A_{q}}{1-q}\right| \frac{2}{m \beta}\right)^{3 / 2} \int x^{1 / 2}(1-\sigma x)^{\frac{q}{1-q}} d x
$$

where $\sigma$ is the signal of $\frac{1-q}{A_{q}}$. It should be noticed that this normalization factor depends only on the temperature, as in BG statistics.

We must calculate the quantity $<U_{i} U_{j}>_{q}$ :

$$
<U_{i} U_{j}>_{q}=\frac{1}{n} \int U_{i} U_{j} F_{q}^{(0)} d^{3} U
$$

$$
=\frac{1}{I} \int_{-\infty}^{+\infty} U_{i} U_{j}\left[1-\frac{1-q}{A_{q}} \beta \frac{m}{2} U^{2}\right]^{\frac{q}{1-q}} d^{3} U
$$

Utilizing parity arguments we can show that this integral will be non-zero only for $i=j$, so we have, changing volume element and the variables as was done in the last calculation we obtain:

$$
<U_{i} U_{j}>_{q}=\frac{\delta_{i j}}{3}\left|\frac{A_{q}}{1-q}\right| \frac{2}{m \beta} \frac{\int x^{3 / 2}(1-\sigma x)^{\frac{q}{1-q}} d x}{\int x^{1 / 2}(1-\sigma x)^{\frac{q}{1-q}} d x}
$$

For $\sigma=+1$, these integrals are made in the interval between 0 and 1 , due to Tsallis cut-off condition, and give the result:

$$
<U_{i} U_{j}>_{q}=\frac{\delta_{i j}}{m \beta}
$$

For $\sigma=-1$, the integrals are calculated between 0 and $+\infty$ and give the same result.

The other expectation value, $\left\langle U_{i} U^{2}>_{q}\right.$ vanishes in this approximation because it is an odd function.

The mass conservation equation is the same in all orders of approximation:

$$
\frac{\partial \rho}{\partial t}+\partial_{i}\left(\rho u_{i}\right)=0
$$

The momentum conservation equation is written as:

$$
\frac{\partial}{\partial t}\left(\rho u_{i}\right)+\partial_{j}\left(\rho u_{i} u_{j}\right)+\partial_{j}\left(\delta_{i j} \frac{\rho}{m \beta}\right)=\rho \frac{F_{i}}{m}
$$

Using the mass equation, we have:

$$
\left[\frac{\partial}{\partial t}+u_{j} \partial_{j}\right] u_{i}+\frac{1}{\rho} \partial_{i}\left(\frac{\rho}{m \beta}\right)=\frac{F_{i}}{m}
$$

The term $\frac{\rho}{m \beta}$ is defined as the pressure of the system:

$$
P=\frac{n}{\beta}
$$

$$
P V=N k_{B} T
$$

We recognize this equation as the equation of state of the ideal gas. As we can see it is independent of $q$.

The energy conservation equation is written as:

$\rho \frac{\partial}{\partial t}\left(\frac{1}{\beta}\right)+\frac{1}{\beta} \frac{\partial \rho}{\partial t}+\rho u_{i} \partial_{i}\left(\frac{1}{\beta}\right)+\frac{1}{\beta} \partial_{i}\left(\rho u_{i}\right)+\frac{2}{3} \delta_{i j} \frac{\rho}{\beta} \partial_{j} u_{i}=0$

Again using the equation for the mass and dividing by $\rho$ :

$$
\left[\frac{\partial}{\partial t}+u_{i} \partial_{i}\right] \frac{1}{\beta}+\frac{2}{3}\left(\partial_{i} u_{i}\right) \frac{1}{\beta}=0
$$

We conclude that, in the zero order approximation, the hydrodynamic equations are q-invariant and we obtained the correct equation of state for the ideal gas. 


\section{The First Order Approximation}

To obtain the fully hydrodynamic equations a solution of Boltzmann transport equation is needed to be used as the distribution function. Since this equation until now was not exactly solved, we made an approximation in the previous section. Now we add to this zero order approximation a correction to it. This procedure consist in writing this exact function as:

$$
F_{1}=F_{q}^{(0)}+F_{q}^{(1)}
$$

where $F_{q}^{(1)}$ is the correction to the zero order approximation. It can be shown that this correction is given by the following relation [10]:

$$
F_{q}^{(1)}=-\tau\left(\frac{\partial}{\partial t}+v_{i} \partial_{i}+\frac{F_{i}}{m} \partial_{v_{i}}\right) F_{q}^{(0)}
$$

where $\tau$ is a parameter of the order of magnitude of the collision time. We must calculate each of the derivatives of $F_{q}{ }^{(0)}$. At first we evaluate the following derivatives. For the density we have:

$$
\begin{gathered}
\frac{\partial F_{q}^{(0)}}{\partial \rho}=\frac{\partial}{\partial \rho}\left[\frac{\rho}{m I} f^{(0)^{\frac{q}{1-q}}}\right] \\
\frac{\partial F_{q}^{(0)}}{\partial \rho}=\frac{F_{q}^{(0)}}{\rho}
\end{gathered}
$$

Second the average velocity:

$$
\begin{aligned}
& \frac{\partial F_{q}{ }^{(0)}}{\partial u_{i}}=\frac{\partial}{\partial u_{i}}\left[\frac{\rho}{m I} f^{(0) \frac{q}{1-q}}\right] \\
& \frac{\partial F_{q}{ }^{(0)}}{\partial u_{i}}=\frac{q}{A_{q}} \beta m U_{i} \frac{F_{q}^{(0)}}{f^{(0)}}
\end{aligned}
$$

Third the temperature:

$$
\begin{gathered}
\frac{\partial F_{q}^{(0)}}{\partial\left(\frac{1}{\beta}\right)}=\frac{\partial}{\partial\left(\frac{1}{\beta}\right)}\left[\frac{\rho}{m I} f^{(0)^{\frac{q}{1-q}}}\right] \\
\frac{\partial F_{q}^{(0)}}{\partial\left(\frac{1}{\beta}\right)}=\frac{q}{A_{q}} \beta^{2} \frac{m}{2} U^{2} \frac{F_{q}^{(0)}}{f^{(0)}}-\frac{3}{2} \beta F_{q}{ }^{(0)}
\end{gathered}
$$

The derivative of the distribution function with respect to the thermal velocity of the molecules is simply the derivative with respect to the average velocity with a changed signal:

$$
\frac{\partial F_{q}^{(0)}}{\partial v_{i}}=-\frac{q}{A_{q}} \beta m U_{i} \frac{F_{q}^{(0)}}{f^{(0)}}
$$

Using these results in the first order approximation function we get:

$$
\begin{array}{r}
F_{q}{ }^{(1)}=-\frac{\tau}{\rho}\left(1-\frac{q}{A_{q}} \frac{1}{f^{(0)}}\right) U_{i} \partial_{i} \rho F_{q}{ }^{(0)} \\
+\tau \frac{q}{A_{q}} \frac{1}{f^{(0)}} \beta m\left(U_{i} U_{j}-\frac{\delta_{i j}}{3} U^{2}\right) \partial_{i} u_{j} F_{q}{ }^{(0)} \\
+\tau \beta\left[\frac{q}{A_{q}} \frac{1}{f^{(0)}}\left(\beta \frac{m}{2} U^{2}-1\right)-\frac{3}{2}\right] U_{i} \partial_{i} \frac{1}{\beta} F_{q}{ }^{(0)}
\end{array}
$$

This function is different from the one calculated by the Maxwell-Boltzmann function [10], it has the explicit dependence on $q$ through the term $\frac{q}{A_{q}} \frac{1}{f^{(0)}}$, which is unity in the extensive limit.

The quantity $<U_{i} U_{j}>_{q}$, in this order of approximation, is given by:

$$
<U_{i} U_{j}>_{q}=\frac{1}{n}\left[\int U_{i} U_{j} F_{q}{ }^{(0)} d^{3} U+\int U_{i} U_{j} F_{q}{ }^{(1)} d^{3} U\right]
$$

The first term is simply $\frac{\delta_{i j}}{m \beta}$. The second term will give non-vanishing contribution only through the second term from equation (28), the others are odd functions:

$$
\begin{aligned}
<U_{i} U_{j}>_{q}=- & \frac{q}{A_{q}} \tau \beta \frac{m}{n} \partial_{l} u_{k}\left[\int U_{i} U_{j} U_{k} U_{l} \frac{F_{q}^{(0)}}{f^{(0)}} d^{3} U-\right. \\
& \left.\frac{\delta_{k l}}{3} \int U_{i} U_{j} U^{2} \frac{F_{q}^{(0)}}{f^{(0)}} d^{3} U\right]
\end{aligned}
$$

This equation is a traceless tensor and it is suffices to calculate any component of it. Putting $i=1$ and $j=2$, we have the result:

$$
\begin{gathered}
<U_{1} U_{2}>_{q}=-\frac{q}{A_{q}} \tau \beta \frac{m}{I} \partial_{l} u_{k} \int U_{1} U_{2} U_{k} U_{l} \\
\times\left[1-\frac{1-q}{A_{q}} \beta \frac{m}{2} U^{2}\right]^{\frac{q}{1-q}-1} d^{3} U
\end{gathered}
$$

This integral is non-zero only for $k=1,2$ and $l=1,2$. We have, writing $U_{1}=U \operatorname{sen} \theta \cos \phi, U_{2}=U \operatorname{sen} \theta \operatorname{sen} \phi$ and changing the volume element to a spherical one:

$$
\begin{aligned}
& <U_{1} U_{2}>_{q}=-\frac{q}{A_{q}} \tau \beta \frac{m}{I}\left(\partial_{1} u_{2}+\partial_{2} u_{1}\right) \\
& \times \int U^{6}\left[1-\frac{1-q}{A_{q}} \beta \frac{m}{2} U^{2}\right]^{\frac{q}{1-q}-1} d U \\
& \quad \times \int_{0}^{\pi} \operatorname{sen}^{5} \theta d \theta \int_{0}^{2 \pi} \operatorname{sen}^{2} \phi \cos ^{2} \phi d \phi
\end{aligned}
$$

Using equations (18) and (19), we have: 


$$
\begin{aligned}
&<U_{1} U_{2}>_{q}=-\frac{q}{A_{q}} \tau \beta \frac{m}{15}\left(\partial_{1} u_{2}+\partial_{2} u_{1}\right)\left(\frac{A_{q}}{1-q}\right)^{2} \\
& \times\left(\frac{2}{m \beta}\right)^{2} \frac{\int x^{5 / 2}(1-\sigma x)^{\frac{q}{1-q}-1} d x}{\int x^{1 / 2}(1-\sigma x)^{\frac{q}{1-q}} d x}
\end{aligned}
$$

For the $\sigma=+1$ case, the above integrals give:

$$
<U_{1} U_{2}>_{q}=-\frac{\tau}{m \beta}\left(\partial_{1} u_{2}+\partial_{2} u_{1}\right)
$$

For the other case, $\sigma=-1$ :

$$
<U_{1} U_{2}>_{q}=-\frac{\tau}{m \beta}\left(\partial_{1} u_{2}+\partial_{2} u_{1}\right)
$$

So the value of $\left\langle U_{i} U_{j}>_{q}\right.$ in this order can be written as:

$$
<U_{i} U_{j}>_{q}=\frac{\delta_{i j}}{m \beta}-\frac{\tau}{m \beta}\left(\partial_{i} u_{j}+\partial_{j} u_{i}-\frac{2}{3} \delta_{i j} \partial_{k} u_{k}\right)
$$

This term doesn't change in this context.

The momentum conservation energy is written as:

$$
\begin{aligned}
& \frac{\partial}{\partial t}\left(\rho u_{i}\right)+\partial_{j}\left[\frac{\rho}{m \beta}-\frac{\tau \rho}{m \beta}\left(\partial_{i} u_{j}+\partial_{j} u_{i}\right.\right. \\
& \left.\left.-\frac{2}{3} \delta_{i j} \partial_{k} u_{k}\right)\right]+\partial_{j}\left(\rho u_{i} u_{j}\right)=\rho \frac{F_{i}}{m}
\end{aligned}
$$

where the term $\frac{\tau \rho}{m \beta}$ is the viscosity coefficient of this system:

$$
\mu=\frac{\tau \rho}{m \beta}
$$

The other expectation value to be calculated is given by, where only the first and third terms of (28) will give nonvanishing contributions:

$$
\begin{gathered}
<U_{i} U^{2}>_{q}=-\frac{\tau}{n} \frac{\delta_{i j}}{3}\left(\int U^{4} P_{q}{ }^{(0)} d^{3} U\right. \\
\left.-\frac{q}{A_{q}} \int U^{4} \frac{P_{q}^{(0)}}{f^{(0)}} d^{3} U\right)+\beta\left(\beta \frac{m}{2} \frac{q}{A_{q}} \int U^{6} \frac{P_{q}^{(0)}}{f^{(0)}} d^{3} U\right. \\
\left.-\frac{q}{A_{q}} \int U^{4} \frac{P_{q}^{(0)}}{f^{(0)}} d^{3} U-\frac{3}{2} \int U^{4} P_{q}^{(0)} d^{3} U\right) \partial_{j} \frac{1}{\beta}
\end{gathered}
$$

Calculating each integral separately, we have:

$$
I_{1}=\int U^{4} F_{q}{ }^{(0)} d^{3} U
$$

This integral is calculated in the same way that the others above, changing the volume elements, using equation (17) and observing the cut-off condition, we obtain:

$$
I_{1}=n \frac{A_{q}}{A_{q}+(1-q)} \frac{15}{(m \beta)^{2}}
$$

The second integral is given by:

$$
I_{2}=\frac{q}{A_{q}} \int U^{4} \frac{F_{q}{ }^{(0)}}{f^{(0)}} d^{3} U
$$

which results in:

$$
I_{2}=n \frac{15}{(m \beta)^{2}}
$$

The last one is given by:

$$
I_{3}=\beta \frac{m}{2} \frac{q}{A_{q}} \int U^{6} \frac{F_{q}^{(0)}}{f^{(0)}} d^{3} U
$$

Resulting in:

$$
I_{3}=n \frac{105}{2(m \beta)^{2}} \frac{A_{q}}{A_{q}+(1-q)}
$$

Using the above results, the expectation value $<U_{i} U^{2}>_{q}$ is given by:

$$
\begin{gathered}
<U_{i} U^{2}>_{q}=\frac{1-q}{A_{q}+(1-q)} \frac{5 \tau}{\rho(m \beta)^{2}} \partial_{i} \rho \\
-\frac{A_{q}-(1-q)}{A_{q}+(1-q)} \frac{5 \tau}{m^{2} \beta} \partial_{i} \frac{1}{\beta}
\end{gathered}
$$

The energy conservation equation is given by:

$$
\begin{gathered}
\frac{3}{2 m} \frac{\partial}{\partial t}\left(\frac{\rho}{\beta}\right)+\frac{3}{2 m} \partial_{i}\left(\frac{\rho}{\beta} u_{i}\right)+\partial_{j} u_{i}\left[\frac{\rho}{m \beta}\right. \\
\left.-\frac{\tau \rho}{m \beta}\left(\partial_{i} u_{j}+\partial_{j} u_{i}-\frac{2}{3} \delta_{i j} \partial_{k} u_{k}\right)\right]+ \\
+\frac{1}{2} \partial_{i}\left[\frac{1-q}{A_{q}+(1-q)} \frac{5 \tau}{(m \beta)^{2}} \partial_{i} \rho\right. \\
\left.-\frac{A_{q}-(1-q)}{A_{q}+(1-q)} \frac{5 \tau \rho}{m^{2} \beta} \partial_{i} \frac{1}{\beta}\right]=0
\end{gathered}
$$

Using the equation for the mass, we have:

$$
\begin{gathered}
\frac{\partial}{\partial t} \frac{1}{\beta}+u_{i} \partial_{i} \frac{1}{\beta}=-\frac{2}{3 \beta} \partial_{j} u_{i}+\frac{2 \tau}{3 \beta}\left(\partial_{i} u_{j}+\partial_{j} u_{i}\right. \\
\left.-\frac{2}{3} \delta_{i j} \partial_{k} u_{k}\right) \partial_{j} u_{i}-\frac{1}{\rho} \partial_{i}\left(K_{q} \partial_{i} \frac{1}{\beta}-\frac{T_{q}}{\rho} \partial_{i} \frac{\rho}{\beta}\right)
\end{gathered}
$$

where: 


$$
K_{q}=\frac{A_{q}}{A_{q}+(1-q)} \frac{5 \tau \rho}{3 m \beta}
$$

is the q-dependent thermal conductivity coefficient. This coefficient, due to its dependence on $q$, can assume positive, $q<1,4$ and $q>1,6$, or negative values, $1,4<$ $q<1,6$. These negative values are non physical. Also we have defined:

$$
T_{q}=\frac{1-q}{A_{q}+(1-q)} \frac{5 \tau \rho}{3 m \beta}
$$

which is the new transport coefficient. This one too can have positive, $q<1$ e $q>1,4$, as well as negative values, $1<q<1,4$. This expression vanishes, properly, in the extensive limit, $q \rightarrow 1$.

The relation between these two coefficients is given by:

$$
\frac{T_{q}}{K_{q}}=\frac{1-q}{A_{q}}
$$

The relation between the thermal conductivity and the viscosity coefficients is given by:

$$
\frac{K_{q}}{\mu}=\frac{A_{q}-(1-q)}{A_{q}+(1-q)} \frac{5}{3}
$$

So the old relation is now $q$ dependent and is equal to the old value in the extensive limit since $A_{q} \rightarrow 1$ when $q \rightarrow 1$.

The coefficients obtained by Boghosian are related to those obtained here through the following relation:

$$
\frac{K_{q}}{K_{B}}=\frac{T_{q}}{T_{B}}=A_{q}=1+(1-q) \frac{3}{2}
$$

\section{CONCLUSIONS}

The hydrodynamic equations obtained here are similar to those obtained by Boghosian 8. The same changes in the energy conservation equation in first order are observed here, only that we have showed that the q-dependence of the thermal conductivity and the new transport coefficients are different.We have showed also, through the derivation of the conservation equations, that the ideal gas state equation is still valid in the generalized statistics context.

As was said there are some differences between our results and the ones obtained by Boghosian \&]. The most important is that these differences in the $q$-dependence of the transport coefficients, in the thermal conductivity and in the new transport coefficient are due the difference in the state equation and in the internal energy for the ideal gas. In our result we do not have intrinsically a $q$-dependence, while in the Boghosian calculation they have a factor $A_{q}=1+(1-q) \frac{3}{2}$. In order to now what result is the correct one is necessary to make some application of these transport equations. In despite of this we believe that our results are better because we obtain the correct state equation (independent of the parameter $q$ ) and we assume the validity of the classical equipartition theorem.

\section{ACKNOWLEDGMENTS}

We would like to thank FUNCAP and CNPq (Brazilian Agencies) for financial supporting and Prof. M. P. Almeida and Evaldo M. F. Curado for enlightening discussions.

[1] C. Tsallis, J. Stat. Phys. 52(1988), 479.

[2] C. Beck, Fractal and Solitons (2001)[condmat/0005408].

[3] A. R. Plastino, A. Plastino, Phys. Lett. A 174(1993), 384.

[4] http://tsallis.cat.cbpf.br/biblio.htm

[5] C. Tsallis, R. S. Mendes, A. R. Plastino, Physica A 261(1998), 534.

[6] S. Abe, S. Martínez, F. Pennini and A. Plastino, Phys. Lett. A (2001) cond-mat/0006109

[7] S. Martinez, F. Nicolás, F. Pennini and A. Plastino, Physica A 286(2000), 489.

[8] B. M. Boghosian, Bras. Jour. Phys. 29(1999), 91.

[9] J. A. S. Lima, R. Silva Jr. and A. R. Plastino, condmat/0101030

[10] K. Huang, "Statistical Mechanics", Wiley, New York, 1987. 